\title{
Effects of Lipophilic Derivatives of L-Ascorbic Acid and Dehydro-L-Ascorbic Acid on the Peroxidation of Linoleic Acid in Neutral Phosphate Buffer Containing Alcohol
}

\author{
Masanosuke TAKAGI, Hiroshi HigASHIOKA, \\ and Naofumi MORITA ${ }^{1}$ \\ Department of Agricultural Chemistry, College of \\ Agriculture, University of Osaka Prefecture, \\ Sakai, Osaka 591, Japan \\ (Received February 19, 1986)
}

\begin{abstract}
Summary 6-O-Palmitoyl-AsA (AP) and -DHA (DHAP) suppressed LA peroxidation considerably in both $10 \%$ and $20 \% \mathrm{EtOH}$ solutions. The duration of the suppression of LA peroxidation was longer with AP than with DHAP. But after the initial suppression of LA peroxidation, both derivatives showed an accelerating effect. 6-O-Acetyl-AsA (Ac-AsA) and -DHA (Ac-DHA) accelerated LA peroxidation from the start of the reaction in $10 \% \mathrm{EtOH}$, but suppressed it notably in $20 \% \mathrm{EtOH}$. 4-Phenyl2,3-dihydroxy-2-buten-4-olide (PDHB) and 4-phenyl-2,3-dioxo-4-butenolide (PDOB) accelerated LA peroxidation in $10 \%$ EtOH. With $20 \%$ EtOH solution, PDHB suppressed LA peroxidation notably, as did AP, but PDOB showed only a short duration (about $1 \mathrm{~h}$ ) of suppression. These results suggest the complexity of LA peroxidation catalyzed by lipophilic AsA or DHA in aqueous solution containing alcohol.

Key Words peroxidation of linoleic acid, lipophilic catalysts, ascorbic acid, dehydroascorbic acid, palmitoyl ascorbate, acyl ascorbate, 4-phenyl2,3-dihydroxy-2-butenolide
\end{abstract}

AsA is widely added to food or food ingredients as a non-nutrient agent to function as a preservative or oxygen acceptor, because of the oxidation-reduction reaction of the ene-diol group of AsA. However, as AsA is easily degraded to DHA in the presence of moisture (I), the role of AsA or DHA on the lipid peroxidation is difficult to understand clearly. We have reported in our previous paper(2) that the effects of acceleration and suppression on the linoleic acid (LA) peroxidation catalyzed by AsA and DHA were related to the state of dispersion of the lipid used.

1 高木正之助, 東岡 洋, 森田尚文 
Since palmitoyl AsA is rather lipophilic compared with AsA, somewhat different results on LA peroxidation are to be expected. In fact, the palmitoyl ester of AsA has been used as a lipophilic antioxidant in food (3-7). Therefore, we tested the effects of 6-O-palmitoyl-AsA (AP) or -DHA (DHAP) on the peroxidation in neutral buffer containing $10 \%$ and $20 \% \mathrm{EtOH}$, in which AsA has the opposite effects of acceleration or suppression on LA peroxidation with different alcohol contents. In this paper, we also describe 6-O-acetyl-AsA (Ac-AsA), -DHA (Ac-DHA), 4phenyl-2,3-dihydroxy-2-buten-4-olide (PDHB), and 4-phenyl-2,3-dioxo-4-butenolide (PDOB) as lipophilic catalysts for LA peroxidation.

\section{MATERIALS AND METHODS}

Materials. LA was purchased from Sigma Chemical Co. and diluted with freshly distilled EtOH to $100 \mathrm{~mm}$ under bubbling with $\mathrm{N}_{2}$ gas. The solution was stored at $-20^{\circ} \mathrm{C}$ until use. AP was obtained from Wako Pure Chemical Industries, Ltd. DHAP was prepared from AP by the method reported previously $(8,9)$. Highly purified water and distilled EtOH were used to remove any contaminant metals. All the buffer solutions used were passed through a short Chelex 100 column(10). AcAsA was prepared from AsA according to a method reported previously (11), and Ac-DHA was prepared from Ac-AsA as mentioned above. Other reagents were of analytical grade.

Analytical methods. To test LA peroxidation, the reaction conditions were the same as reported previously $(8,9)$. The reaction mixture for $10 \mathrm{mM} \mathrm{LA}$ and $20 \%$ EtOH solution was prepared by mixing $0.5 \mathrm{ml}$ of $100 \mathrm{~mm} \mathrm{LA} \mathrm{in} \mathrm{EtOH,} 0.5 \mathrm{ml}$ of EtOH, $4 \mathrm{ml}$ of phosphate buffer $(0.1 \mathrm{M}, \mathrm{pH} 6.8)$, and $0.1 \mathrm{ml}$ of the material to be tested $(180 \mu \mathrm{M})$. The reaction mixture was adjusted to $\mathrm{pH} 7$ and incubated at $37^{\circ} \mathrm{C}$ with shaking. To vary the EtOH content, the $\mathrm{pH}$ of the buffer solution was first adjusted to 6.9 for $10 \% \mathrm{EtOH}$ and to 6.8 for $20 \% \mathrm{EtOH}$, so that the final $\mathrm{pH}$ was 7.0. Consequently, the molarity of the buffer was $90 \mathrm{~mm}$ for $10 \% \mathrm{EtOH}$ and $80 \mathrm{~mm}$ for $20 \%$ EtOH. A sample $(0.3 \mathrm{ml})$ of the reaction mixture was withdrawn at specified times and $3 \mathrm{ml}$ of $60 \%$ EtOH aq. solution containing EDTA ( $200 \mathrm{ppm}$ ) was added. The optical densities at $233 \mathrm{~nm}$ and $265 \mathrm{~nm}$ were used for the measurement of the LA hydroperoxide formed and the AsA remaining, respectively.

\section{RESULTS AND DISCUSSION}

\section{Effects of $A P$ and $D H A P$ on the LA peroxidation}

Figure 1 shows the results of LA peroxidation catalyzed by the addition of AP or DHAP. AP suppressed the LA peroxidation in both $10 \%$ and $20 \% \mathrm{EtOH}$. When the $A_{265}$ due to AsA disappeared after several hours of incubation, the amounts of LA hydroperoxide started to increase gradually. Notable suppression of LA peroxidation catalyzed by AP was observed in both $10 \%$ and $20 \%$ EtOH solutions, as compared with control values without catalysts shown in column $\mathrm{C}$ in Fig. 1. 


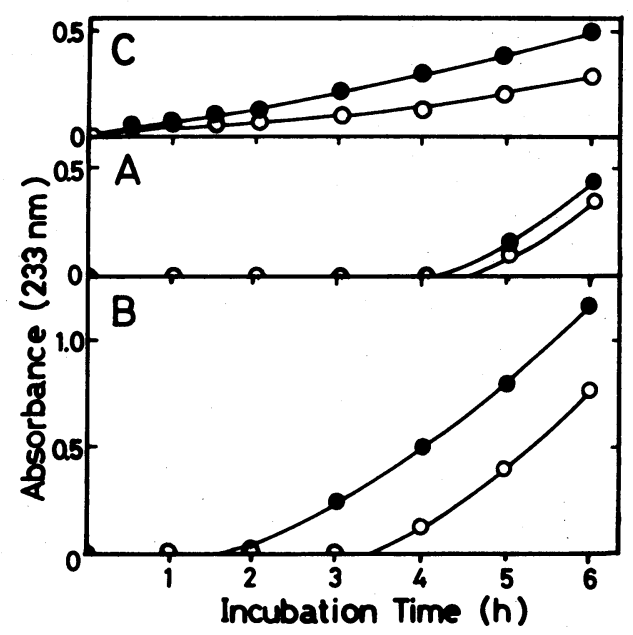

Fig. 1. Peroxidation of linoleate catalyzed by AP (A) or DHAP (B). The reaction mixture contained $10 \mathrm{mM} \mathrm{LA}, 180 \mu \mathrm{M}$ of catalyst and $10 \% \mathrm{EtOH}(\bullet)$ or $20 \% \mathrm{EtOH}$ $(O)$ in phosphate buffer. A sample $(0.3 \mathrm{ml})$ of the reaction mixture was withdrawn and added to $3 \mathrm{ml}$ of $60 \%$ EtOH solution containing EDTA (200 ppm), and the UV absorption spectra of the solution was measured. C shows the control values without AP or DHAP.

However, the duration of the suppression was longer in $20 \% \mathrm{EtOH}$ than in $10 \%$ EtOH. After the apparently perfect suppression, peroxidation began to proceed more quickly with both AP and DHAP.

The rates of LA peroxidation catalyzed by DHAP in $10 \%$ and $20 \% \mathrm{EtOH}$ solutions were similar to those catalyzed by AP, but the duration of the suppression of LA peroxidation by DHAP was somewhat shorter than that by AP. Furthermore, in the system with $10 \% \mathrm{EtOH}$ a rather strong suppression of LA peroxidation was observed even in the case of DHAP; this is quite a different result from the LA peroxidation catalyzed by AsA in 10\% EtOH.

\section{Effects of Ac-AsA and Ac-DHA on the LA peroxidation}

The effects of Ac-AsA and Ac-DHA on the peroxidation of LA were examined as shown in Fig. 2. Addition of Ac-AsA caused only an acceleration of LA peroxidation in $10 \% \mathrm{EtOH}$. With $20 \% \mathrm{EtOH}$ solution, the amounts of LA hydroperoxide increased gradually after the complete disappearance of the absorption peak due to AsA (data not shown). That is, Ac-AsA suppresses LA peroxidation in $20 \%$ EtOH solution for several hours, then accelerates it after the consumption of the Ac-AsA in the reaction mixture.

Addition of Ac-DHA to the reaction mixture showed only an acceleration in $10 \%$ EtOH solution, but both suppression and acceleration of LA peroxidation were seen in $20 \%$ EtOH solution, similar to AP. The pattern of acceleration of LA 


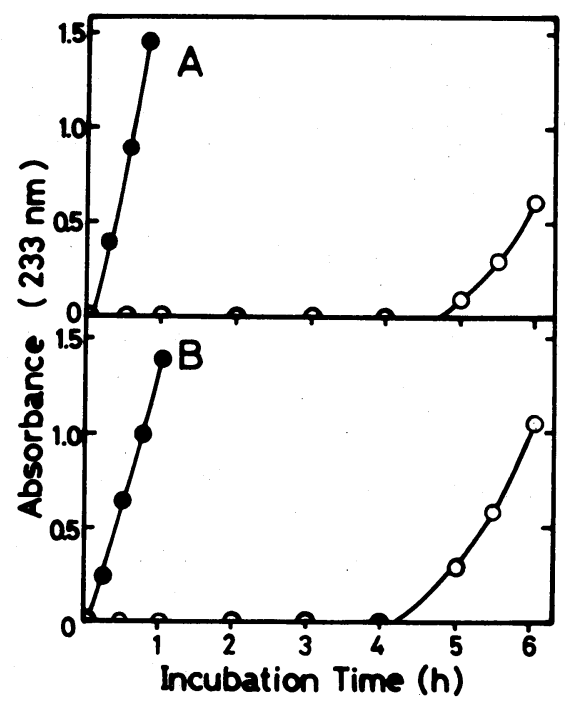

Fig. 2. Peroxidation of linoleate catalyzed by Ac-AsA (A) or Ac-DHA (B). Experimental conditions were the same as for Fig. 1. @, 10\% EtOH; O, 20\% EtOH.

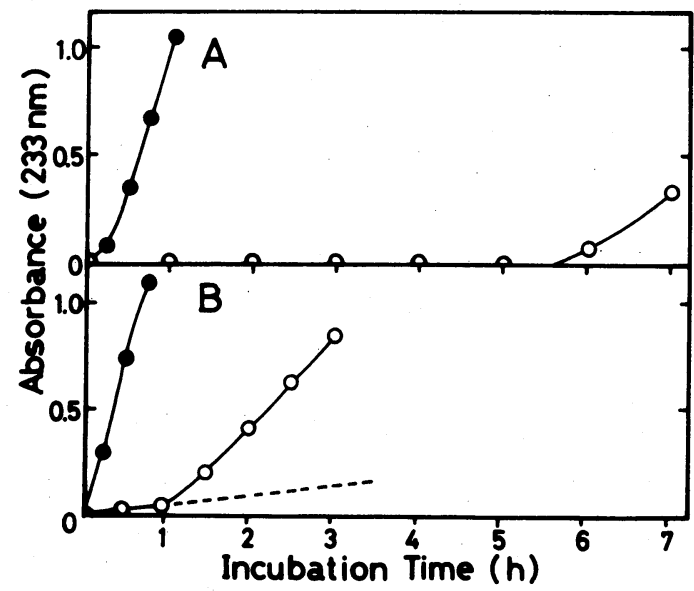

Fig. 3. Peroxidation of linoleate catalyzed by PDHB (A) or PDOB (B). Experimental details were the same as for Fig. 1., $10 \% \mathrm{EtOH} ; \mathrm{O}, 20 \% \mathrm{EtOH}$. The broken line shows the control values in $20 \%$ EtOH solution.

peroxidation catalyzed by Ac-AsA in $10 \%$ EtOH was similar to that catalyzed by Ac-DHA. Likewise, Ac-AsA and Ac-DHA in $20 \%$ EtOH showed almost the same pattern of suppression, followed by acceleration of LA peroxidation.

In the $20 \% \mathrm{EtOH}, \mathrm{Ac}-\mathrm{DHA}$ had almost the same duration of suppression of LA peroxidation as Ac-AsA, then acceleration of LA peroxidation was seen. 
Furthermore, the degree and the rate of acceleration catalyzed by Ac-AsA were almost the same as those catalyzed by Ac-DHA.

\section{Effects of $P D H B$ and $P D O B$ on $L A$ peroxidation}

Figure 3 shows the results of LA peroxidation catalyzed by PDHB in the systems with $10 \%$ and $20 \% \mathrm{EtOH}$. Addition of PDOB to the reaction mixture containing $10 \%$ EtOH solution only accelerated LA peroxidation from the beginning of the reaction, but in the system with $20 \% \mathrm{EtOH}$ solution, a prominent suppression of the LA peroxidation which lasted more than $5 \mathrm{~h}$ was observed, which then changed to an acceleration like AP.

Addition of PDOB accelerated the LA peroxidation in the system with $10 \%$ EtOH solution from the beginning of the reaction, but in the system with $20 \%$ EtOH solution, a suppression of LA peroxidation for $1 \mathrm{~h}$, followed by a gradual acceleration of LA peroxidation, was observed.

Based on these results, we propose that 6-O-acetyl- and phenyl-derivatives of AsA or DHA have almost the same effects on the peroxidation of LA. Furthermore, these two derivatives of AsA or DHA accelerate LA peroxidation in the $10 \% \mathrm{EtOH}$ solution, just like that induced by AsA or DHA alone. On the other hand, in the system with $20 \%$ EtOH solution, the type of LA peroxidation catalyzed by $6-O$ acetyl- and phenyl-derivatives of AsA or DHA is similar to those catalyzed by AP or DHAP, which suppress the LA peroxidation notably for several hours, followed by a gradual acceleration.

These results suggest that the solubilities of the catalysts in the reaction mixture are very important for the peroxidation of LA. Considering the results of the two opposite effects on LA peroxidation, the acceleration and suppression are probably dependent on the dispersion of LA(12). Therefore, the effects of AsA on LA peroxidation are greatly influenced by the partition of AsA or DHA into the water layer or oil layer in the oil-water dispersion system of the reaction mixture (2).

Finally, some comments on the appearance of the reaction mixtures are necessary. Colloidal mixtures of LA and additives in the buffer solutions had considerably different appearances, suggesting the different miscibility of each component in the mixture. The factors of partition of each component in the oil and water phases must be estimated, if we are to discuss this phenomenon more quantitatively. To eliminate this factor, we used lipophilic derivatives instead of pure AsA and DHA. However, although some new information on AP and DHAP was obtained as described above, little additional understanding of lipophilic derivatives of AsA and DHA was gained, as compared with reactions catalyzed by AsA or DHA without a lipophilic moiety. This suggests the complexity of the acceleration and suppression of LA peroxidation catalyzed by AsA or DHA or its lipophilic derivatives in an aqueous solution containing other solvents.

We express our sincere thanks to Dr. Y. Tsujimoto, Osaka City Institute of Public Health and Environmental Sciences, for the kind gift of 4-phenyl-2,3-dihydroxy-2-buten-4- 
olide and its derivative.

\section{REFERENCES}

1) Nomura, D., and Ohmura, H. (1969): Chemie der Reductone, Uchida Rokakuho Shinsha, Tokyo (in Japanese), p. 103.

2) Takagi, M., Higashioka, H., Tamura, K., and Morita, N. (1986): Effects of L-ascorbic acid and dehydro-L-ascorbic acid on the peroxidation of linoleic acid in neutral phosphate buffer containing alcohol. Agric. Biol. Chem., 50, 41-47.

3) Pongracz, G. (1973): Antioxidant mixtures for use in food. Int. J. Vitam. Nutr. Res., 43, 517-525.

4) Johnson, F. C., and Sinclair, H. M. (1979): The antioxidant vitamins. CRC Crit. Rev. Food Sci. Nutr., 11, 210-309.

5) Court, W. M. (1974): Antioxidant activity of tocopherols, ascorbyl palmitate and ascorbic acid on their mode of action. J. Am. Oil Chem. Soc., 51, 321-325.

6) Ayano, Y., Furuhashi, T., Watanabe, Y., and Motohashi, K. (1977): Effects of additions of L-ascorbyl stearate on the antioxidative activity of $\alpha$-tocopherol. Nippon Shokuhin Kogyo Gakkaishi (in Japanese), 24, 372-374.

7) Hoseney, R. C., Seib, P. A., and Doyoe, C. W. (1977): Use of salts of 6-acyl esters of Lascorbic acid and D-isoascorbic acid in bread making. Cereal Chem., 54, 1062-1069.

8) Ohmori, M., and Takagi, M. (1978): A facile preparation of dehydro-L-ascorbic acidmethanol solution and its stability. Agric. Biol. Chem., 42, 173-174.

9) Ohmori, M., Higashioka, H., and Takagi, M. (1983): Pure dehydro-L-ascorbic acid prepared by $\mathrm{O}_{2}$-oxidation of L-ascorbic acid with active charcoal as catalyst. Agric. Biol. Chem., 47, 607-608.

10) Agarwal, M., Bennett, R. S., Stump, I. G., and D'Auria, J. M. (1975): Analysis of urine for trace elements by energy dispersive chelating resin. Anal. Chem., 47, 924-927.

11) Tanaka, H., and Yamamoto, R. (1966): Pharmaceutical studies on ascorbic acid derivatives. 1. Synthesis of esters of ascorbic acid and their physicochemical properties. Yakugaku Zasshi (in Japanese), 86, 376-383.

12) Yau, T. M., and Mencl, J. (1981): A study of the peroxidation of fatty acid micelles promoted by ionizing radiation, hydrogen peroxide, and ascorbate. Int. J. Radiat. Biol., 40, 47-61. 